\title{
SOME RANDOM FIXED POINT THEOREMS WITH PPF DEPENDENCE FOR WEAKLY CONTRACTIVE RANDOM OPERATOR
}

\author{
PHAM THE ANH
}

Abstract. In this paper, the fixed point theorem for weakly contraction proved by Rhoades [Nonlinear Anal. 47 (4) (2001), 2683-2693] will be extended for the random case with PPF dependence. By the constructive method, the result is proved and an application of this theorem for random equation is given.

Mathematics subject classification (2010): 60B11, 60G57, 47H10, 34K10.

Keywords and phrases: random fixed point theorem, contractive mapping, weakly contractive mapping, PPF dependence.

\section{REFERENCES}

[1] S.R. Bernfeld, V. Lakshmikantham, Y.M. Reddy, Fixed point theorems of operators with PPF dependence in Banach spaces, Applicable Anal., 6, 4 (1977), 271-280.

[2] I. BEG, M. ABBAS, Iterative procedures for solutions of random operator equations in Banach spaces, J. Math. Anal. Appl., 315, 1 (2006), 181-201.

[3] A.T. Bharucha-ReID, Fixed point theorems in probabilistic analysis, Bull. Amer. Math. Soc., 82, 5 (1976), 641-657.

[4] B.C. DHAGE, Some basic random fixed point theorems with PPF dependence and functional random differential equations, Differ. Equ. Appl., 4, 2 (2012), 181-195.

[5] C.J. Himmelberg, Measurable relations, Fund. Math., 87 (1975), 53-72.

[6] B.E. RHoAdes, Some theorems on weakly contractive maps, Nonlinear Anal., 47, 4 (2001), 2683 2693.

[7] K.K. Tan, X.Z. Yuan, On deterministic and random fixed points, Proc. Amer. Math. Soc., 119, 3 (1993), 849-856.

[8] S. LI, X. XIAO, L. LI, J. Lv, Random approximation with weak contraction random operators and a random fixed point theorem for nonexpansive random self-mappings, J. Inequal. Appl., 2012, 2012:16, $7 \mathrm{pp}$. 